\title{
TRIM37 targets AKT in the growth of lung cancer cells
}

This article was published in the following Dove Press journal:

OncoTargets and Therapy

\begin{abstract}
Shumin Dong'
Xueqin Pang ${ }^{2}$

Haijun Sun ${ }^{3}$

Chunluan Yuan ${ }^{4}$

Chuanyong $\mathrm{Mu}^{5}$

Shiying Zheng ${ }^{6}$

'Department of Thoracic Surgery, The First Affiliated Hospital of Soochow University, The First People's Hospital of Lianyungang City, Lianyungang, Jiangsu 222000, People's Republic of China; ${ }^{2}$ Department of Gastroenterology, The First Affiliated Hospital of Soochow University, Suzhou, Jiangsu 215006, People's Republic of China; ${ }^{3}$ Department of Thoracic Surgery, The First People's Hospital of Lianyungang City, Lianyungang, Jiangsu 222000, People's Republic of China; ${ }^{4}$ Department of Oncology, The First People's Hospital of Lianyungang City, Lianyungang, Jiangsu 222000, People's Republic of China; ${ }^{5}$ Department of Respiratory Medicine, The First Affiliated Hospital of Soochow University, Suzhou, Jiangsu 2I 5006, People's Republic of China; ${ }^{6}$ Department of Thoracic Surgery, The First Affiliated Hospital of Soochow University, Suzhou, Jiangsu 2I 5006, People's Republic of China
\end{abstract}

Correspondence: Chuanyong Mu Department of Respiratory Medicine, The First Affiliated Hospital of Soochow University, Shizi Street No 188, Suzhou, Jiangsu 215006, People's Republic of China

Emailmu_chuan_yong@163.com

Shiying Zheng

Department of Thoracic Surgery, The

First Affiliated Hospital of Soochow University, Shizi Street No 188, Suzhou, Jiangsu 215006, People's Republic of China

Email syzheng571025@163.com
Background: TRIM37 is an ubiquitin E3 ligase. Growing evidence has demonstrated the high value of TRIM37 as a potential biomarker for diagnosis of certain cancers. However, the biological function of TRIM37 in lung cancer is still unknown.

Materials and methods: In order to gain a deep insight into the function of TRIM37 in lung cancer cells, in the present study lentiviral vector was used to mediate RNA interference and overexpression of TRIM37 in lung cancer cells (H292, H358, and H1299). In addition, a specific AKT inhibitor LY294002 was utilized to examine the correlation between the expression of TRIM37 and AKT.

Results: TRIM37 acts as a positive regulator of cell proliferation in lung cancer cells. Moreover, cell apoptosis analyses showed the antiapoptosis function of TRIM37, which was mainly dependent on the regulation of BCL2 and BAX. Our results also indicated that AKT might be a target of TRIM37 in lung cancer cells.

Conclusion: This research not only helps in understanding the molecular mechanisms of TRIM37 in detail but also provides evidence to develop novel biomarkers for lung cancer diagnosis.

Keywords: TRIM37, cell proliferation, apoptosis, BCL2, BAX, AKT

\section{Introduction}

Lung cancer is one of the most commonly diagnosed cancers worldwide and has been ranked as the top killer cancer all over the world. ${ }^{1,2}$ Though the traditional treatment (resection and radiotherapy) can contribute to fighting against the recurrence of lung cancer, the outcome usually remains poor. Therefore, gaining a deep insight into the molecular mechanism of lung cancer would help to develop new biomarkers and therapy for this disease.

TRIM37 is one of the members of TRIM family, which is mapped on chromosome 17q22-23. ${ }^{3}$ TRIM37 serves as an ubiquitin E3 ligase and plays an essential role in oncogenesis. A previous report has shown that TRIM37 is upregulated in human colorectal cancer (CRC) and contributes to the development of CRC. ${ }^{4}$ Moreover, the expression of TRIM37 is significantly upregulated in pancreatic cancerous tissues, which indicates the oncogenic role of TRIM37 in pancreatic cancer. ${ }^{5}$ In addition, high expression of TRIM37 is observed in osteosarcoma. ${ }^{6}$ Growing evidence has indicated that TRIM37 participates in the tumorigenesis of several cancers. However, the biological function of TRIM37 in lung cancer is less well understood.

BCL2 mediates the intrinsic apoptosis pathway. A previous report has shown that BCL2 is also essential for cell cycle progression. ${ }^{7}$ The dysregulation of BCL2 not only damages normal development but also contributes to tumor progression. ${ }^{8}$ The high level of BCL2 suppresses apoptosis in human lung cancer cells, which serves as the antiapoptotic factor in this cancer. ${ }^{9}$ In addition, BAX belongs to the BCL2 family, which 
functions as proapoptotic marker. ${ }^{10}$ Therefore, the occurrence of cell apoptosis depends on the ratio of BAX/BCL2. Dysregulation of the ratio of BAX/BCL2 may lead to carcinogenesis. Taken together, BCL2 family members have potential value as critical targets in lung cancer treatment. ${ }^{11,12}$

The AKT pathway plays an essential role in various biological functions, which is commonly abnormal in human cancer. ${ }^{13}$ The activity of AKT protein relies on its phosphorylation. A previous report has indicated that the AKT phosphorylation ( $\mathrm{p}$-AKT) on Thr308 correlates with the activity of AKT protein in human non-small-cell lung cancer. ${ }^{14}$ The $\mathrm{PI} 3 \mathrm{~K} / \mathrm{AKT}$ pathway is involved in cell cycle and apoptosis of lung cancer cells. ${ }^{15}$ Some evidence has demonstrated that the major function of TRIM37 in glioma cells is the inactivation of PI3K/AKT signaling pathway. ${ }^{16}$ Therefore, further studies are needed to examine the relationship between TRIM37 and AKT in lung cancer cells.

In the present study, we systematically analyzed the function of TRIM37 and its possible targets in lung cancer cells. RNA interference was used to knockdown the expression of TRIM37 in lung cancer cells, while lentiviral vector was used to mediate overexpression of TRIM37. Our data provide not only a deep insight into the molecular mechanisms of TRIM37 but also evidence to develop novel biomarkers for lung cancer.

\section{Materials and methods}

\section{Tissue specimens and cell culture}

A total of 30 pairs of lung cancer tissues and adjacent noncancerous tissues were obtained from patients at the First Affiliated Hospital of Soochow University. Samples were snap-frozen in liquid nitrogen and stored at $-80^{\circ} \mathrm{C}$ for further analysis. All patients provided written informed consent. This research was approved by the independent ethics committee of the First Affiliated Hospital of Soochow University and was carried out in accordance with the Declaration of Helsinki.

All the cell lines (A549, H1299, H1975, H358, H292, and $16 \mathrm{HBE}$ ) used in this study were obtained from the cell bank of Shanghai Institute for Biological Sciences (Shanghai, People's Republic of China). FBS (10\%; Thermo Fisher Scientific, Waltham, MA, USA) was mixed into all culture media along with $2 \mathrm{mM}$ L-glutamine and $1 \%$ penicillin/ streptomycin (Solarbio, Beijing, People's Republic of China). Cells were grown in DMEM (TrueLine, Nashville, TN, USA) and were incubated in a $5 \% \mathrm{CO}_{2}$ atmosphere at $37^{\circ} \mathrm{C}$.

\section{Immunohistochemistry}

In brief, all tissue samples were fixed on $10 \%$ formalin and embedded with paraffin. A set of xylene baths and graded alcohols were used to deparaffinize and rehydrate samples. Antigen retrieval was carried out through steam heating for 15 minutes in $0.01 \mathrm{M}$ citrate buffer at $\mathrm{pH} 6.0$, and slides were rinsed three times with PBS $(0.02 \mathrm{M})$ after natural cooling. Then, the activity of endogenous peroxidase was suppressed using Tris-buffered saline with Tween 20 containing 3\% hydrogen peroxide for 10 minutes. The sections were incubated with a primary antibody (TRIM37; Novus, St Charles, MO, USA) overnight at $4^{\circ} \mathrm{C}$ followed by biotin-labeled secondary antibody (Longislandbio, Shanghai, People's Republic of China) at room temperature for half an hour. Then, hematoxylin was used for nuclear counterstaining after slides were reacted with diaminobenzidine substrate.

\section{RNA isolation and real-time PCR}

Total RNA from all samples was extracted using TRIzol reagent (Thermo Fisher Scientific). After that, cDNA synthesis kit (Thermo Fisher Scientific) was used to synthesize cDNA according to the instruction of the manufacturer. The conditions of real-time PCR were as follows: $95^{\circ} \mathrm{C}$ for 10 minutes followed by 40 cycles of $95^{\circ} \mathrm{C}$ for 15 seconds and $60^{\circ} \mathrm{C}$ for 45 seconds. The expression was normalized to that of GAPDH. The relative gene expression was calculated by the $2^{-\Delta \Delta \mathrm{Ct}}$ method. All data represented the average of three replicates. The primer sequences used in this study are listed in the Supplementary materials.

\section{Lentiviral vector-mediated RNA interference and overexpression of TRIM37}

Lentiviral plasmids (pLKO.1) containing three shRNAs directed to various regions of human TRIM37 (NM_015294.5) and a negative control shRNA (siNC) were synthesized (Major, Shanghai, People's Republic of China), respectively. Lentiviral plasmid (pLVX-puro) containing the full length of human TRIM37 cDNA sequence and the mock plasmid served as the negative control (oeNC). All of them were transiently transfected into lung cancer cells as indicated above using Lipofectamine 2000 (Thermo Fisher Scientific) according to the instruction of the manufacturer. Experiments were performed 48 hours after transfection. Detailed sequence information about siTRIM37s is provided in Table $\mathrm{S} 1$.

\section{Western blot}

Whole-protein lysates were extracted from indicated cells (H292, H358, and H1299) using RIPA lysis buffer (JRDUN, Shanghai, People's Republic of China) with EDTA-free 
protease inhibitor cocktail (Roche, Basel, Switzerland). The protein concentration was estimated using an enhanced BCA protein assay kit (Thermo Fisher Scientific). An equal amount of total protein $(25 \mu \mathrm{g})$ was fractionated on a $10 \%$ SDS-PAGE gel. Then, the gel was transferred to a nitrocellulose membrane (EMD Millipore, Billerica, MA, USA) overnight. After being blocked with 5\% nonfat dry milk for 1 hour at room temperature, the membrane was probed at $4^{\circ} \mathrm{C}$ overnight with primary antibodies followed by secondary antibody anti-mouse $\operatorname{IgG}(1: 1,000$; Beyotime, Haimen, People's Republic of China) for 1 hour at $37^{\circ} \mathrm{C}$. An enhanced chemiluminescence system (Tanon, Shanghai, People's Republic of China) was used for detecting protein expression value. The detailed information of primary antibodies is provided in Table $\mathrm{S} 2$.

\section{Cell proliferation assay}

Cell Counting Kit-8 (CCK-8) assay (SAB, College Park, MD, USA) was performed to examine cell proliferation according to the protocol of the manufacturer. Briefly, cells transfected as indicated were seeded in 96-well plates and cultured for $0,24,48$, and 72 hours. CCK-8 solution (1:10) was mixed to each well and incubated for 1 hour. A microplate reader (Pulangxin, Beijing, People's Republic of China) was used to measure the OD values at a wavelength of $450 \mathrm{~nm}$. Independent triplicate experiments were carried out for each time point.

\section{Cell apoptosis assay}

In brief, H292, H358, and H1299 cells were collected and stained with Annexin V-fluorescein isothiocyanate apoptosis detection kit (Beyotime) according to the instructions of the manufacturer at 48 hours after viral infection. A flow cytometer (BD, Franklin Lakes, NJ, USA) was used to determine cell apoptosis.

\section{Migration assay}

Briefly, the indicated TRIM37 siRNA cells were serumstarved for 24 hours followed by seeding in the upper chamber, while the medium supplemented with 30\% FBS (Thermo Fisher Scientific) was placed in the lower chamber. After 24 hours of incubation, cells on the upper side of the filters were removed and the remaining cells were fixed in $4 \%$ formaldehyde and stained with $0.01 \%$ crystal violet (Solarbio). Then, cells in the lower chamber were stained with crystal violet and counted under a microscope at $\times 200$ magnification (Caikon, Shanghai, People's Republic of
China). All the procedures were performed using Boyden chambers (Costar; Corning, Corning, NY, USA).

\section{Invasion assay}

This assay was also performed using Boyden chambers containing a polycarbonate filter coated with Matrigel on the upper surface. The procedure was performed as indicated above.

\section{Xenograft model}

This assay was carried out according to the institute's guidelines for animal experiments and was approved by the independent ethics committee of the First Affiliated Hospital of Soochow University, People's Republic of China, and all animals were treated in accordance with the Institutional Animal Care and Use Committee (IACUC) guidelines. An equal number of siNC- and siTRIM37-transfected H358 cells $\left(n=2 \times 10^{6}\right)$ were subcutaneously injected into the right flank of 4- to 6-week-old nude mice ( $\mathrm{n}=26$ for each group; Shanghai Laboratory Animal Company, Shanghai, People's Republic of China). The length and width of the tumor were examined every 3 days for 33 days after injection. The volume of the tumor was counted as length $\times$ (width $2 / 2$ ). Six weeks after injection, six mice from the siNC- and siTRIM37injected groups were sacrificed by cervical dislocation and tumor tissues were fixed in $4 \%$ formalin for further analysis. A total of 20 nude mice in each injected group as indicated were used to measure mouse survival rate. Mouse survival was monitored daily for a total of 100 days.

\section{Statistical analysis}

GraphPad Prism software Version 7.0 (GraphPad Software, Inc., La Jolla, CA, USA) was utilized for statistical analyses. Data were expressed as mean \pm SD of at least three samples. Statistical significance was determined by one-way ANOVA for multiple comparisons. A $P$-value $<0.05$ was considered to indicate statistical significance.

\section{Results}

\section{The level of TRIM37 was upregulated in lung cancer tissues and cell lines}

The mRNA expression of TRIM37 was examined by quantitative real-time PCR in 30 pairs of lung cancer samples and matched para-cancerous tissues. In addition, Western blot was utilized to examine the protein level of TRIM37 in eight pairs of lung cancer samples and matched normal tissues. As shown in Figure 1A and B, both the mRNA and protein levels of TRIM37 in lung cancer tissues were significantly 


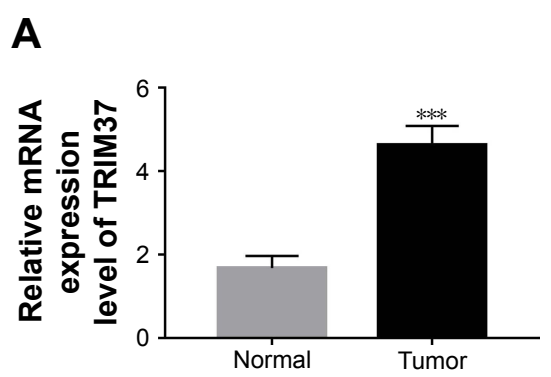

C

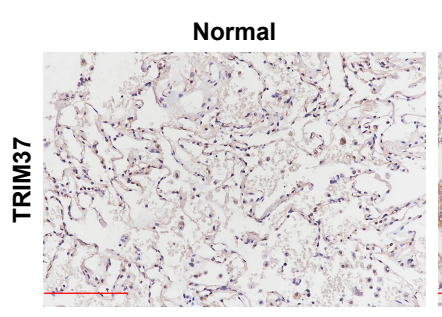

B

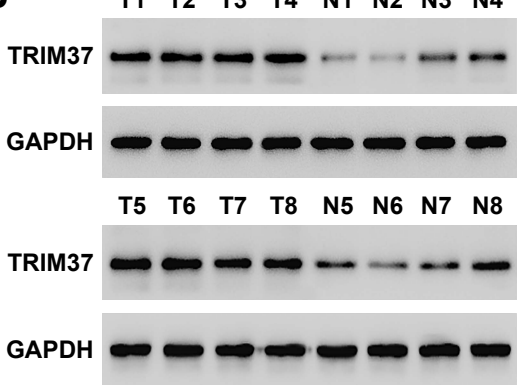

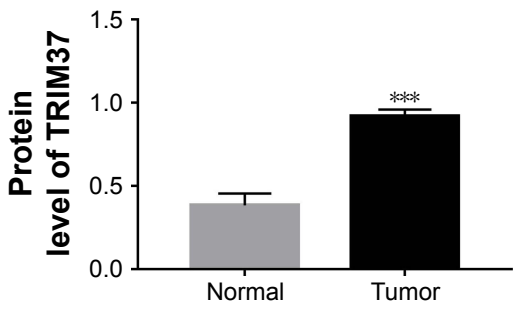

D

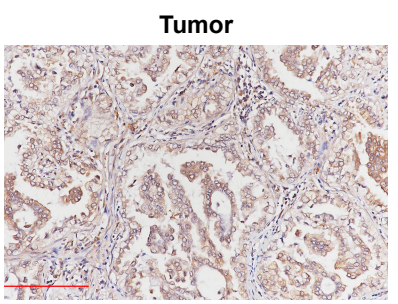

$\times 200$

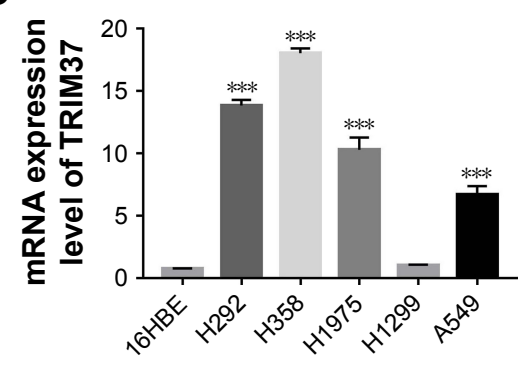

$\mathbf{E}$

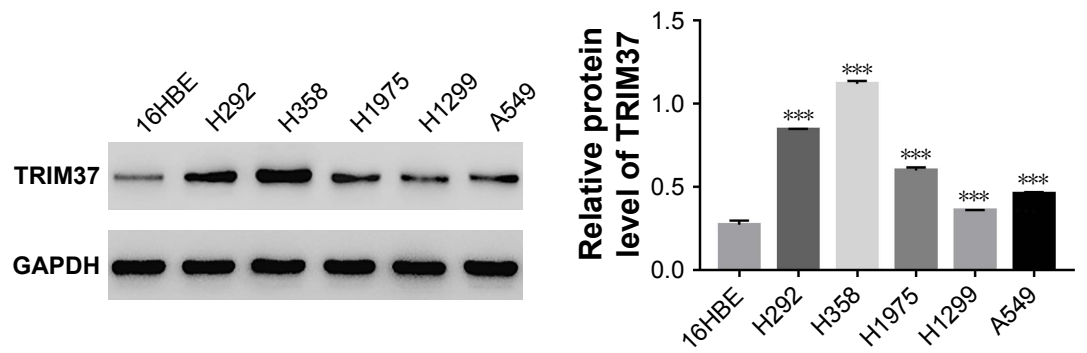

Figure I Expression of TRIM37 in lung cancer tissues and cell lines.

Notes: (A) The mRNA expression of TRIM37 in lung cancer and matched para-cancerous tissues ( $\mathrm{n}=30$ for each group). $* * * P<0.00 \mathrm{I}$ vs normal. (B) The protein level of TRIM37 in lung cancer and matched para-cancerous tissues ( $n=8$ for each group). $* * * P<0.00$ I vs normal. (C) The protein level of TRIM 37 analyzed by immunohistochemistry in lung cancer and matched para-cancerous tissues. (D and $\mathbf{E})$ The mRNA and protein expression level of TRIM 37 in different lung cancer cell lines, respectively. $* * * P<0.00 I$ vs $16 \mathrm{HBE}$ cells.

upregulated as compared with normal tissues. Moreover, immunohistochemistry was used to further investigate the protein level of TRIM37 in lung cancer and normal tissues. The results also suggested that TRIM37 was upregulated in tumor tissues (Figure 1C). Then, the mRNA and protein expression level of TRIM37 was detected in five lung cancer cell lines, including H292, H358, H1299, A549, and H1975, using a human bronchial epithelial cell line (16HBE) as normal control. As shown in Figure 1D and E, the mRNA and protein expression levels of TRIM37 were significantly upregulated in lung cancer cell lines, especially in H292 and H358 cells, compared with 16HBE cells. On the other hand, the H1299 cells showed relatively lower level of TRIM37 than other lung cancer cell lines. Based on these results, H292, H358, and H1299 cell lines were chosen for further study.

\section{Knockdown and overexpression of TRIM37 in lung cancer cells}

To silence the expression of TRIM37, three shRNAs targeting human TRIM37 gene (siTRIM37; RNAi1-1, RNAi1-2, RNAil-3) and a negative control shRNA (siNC) were synthesized and transfected to H292 and H358 cell lines, respectively. The untreated cells acted as blank control (BLANK). As shown in Figure 2A and B, all the TRIM37siRNAs were able to strongly reduce endogenous TRIM37. Cells transfected with RNAil-1 and RNAi1-2 showed lower TRIM37 level than RNAi1-3. Therefore, RNAi1-1 and RNAi1-2 were chosen for further study. In addition, H1299 cells were transfected with a plasmid overexpressing TRIM37 (oeTRIM37) and a mock plasmid (oeNC), respectively. As shown by the TRIM37 mRNA and protein levels in Figure 2C and D, a remarkable overexpression 

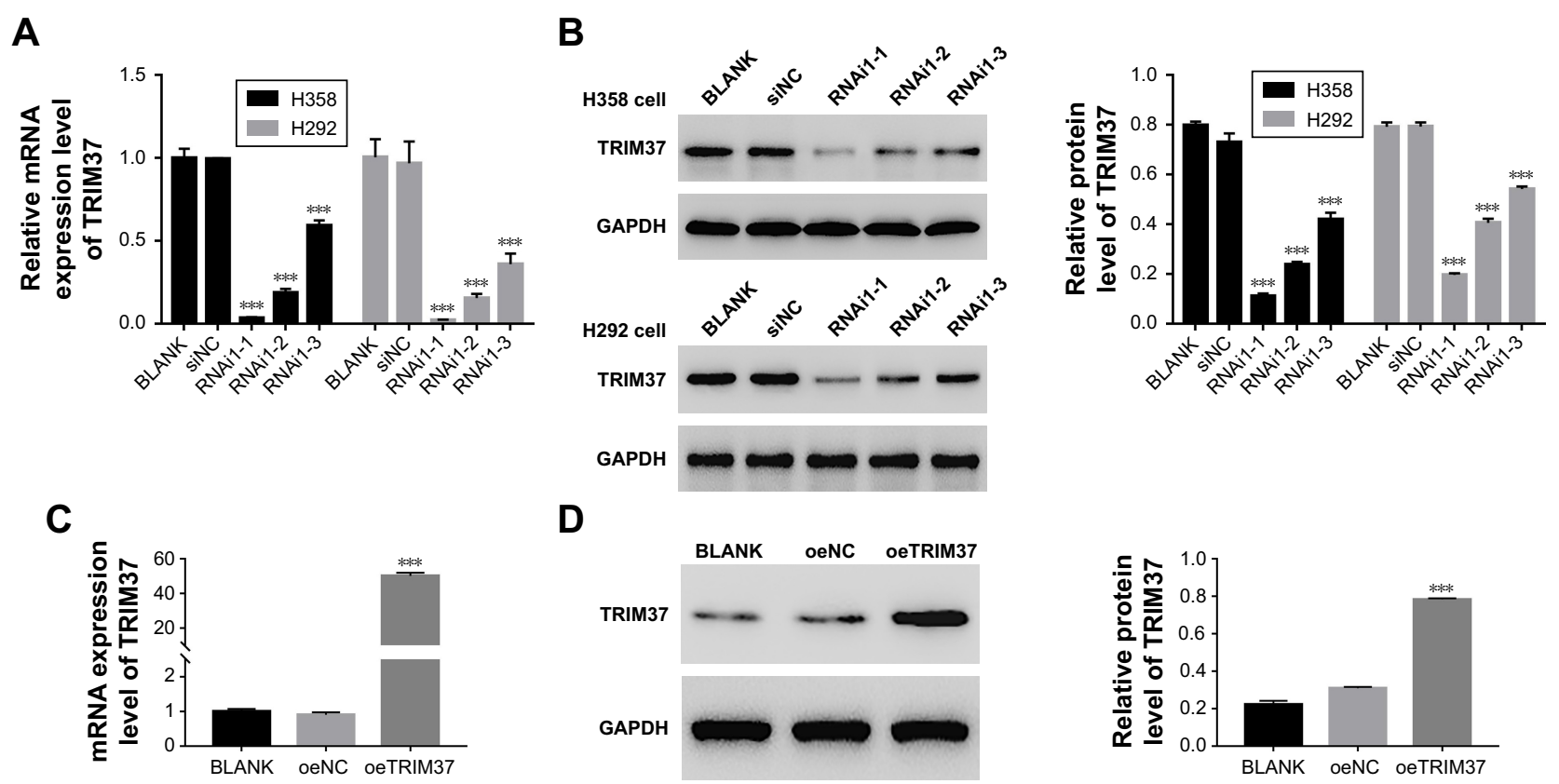

Figure 2 Knockdown and overexpression of TRIM37 in lung cancer cell lines.

Notes: (A and B) The mRNA and protein level of TRIM37 after transfection of siRNAs into $\mathrm{H} 358$ and $\mathrm{H} 292$ cells, respectively. $* * * P<0.00 \mathrm{I}$ vs siNC. (C and D) The mRNA and protein level of TRIM37 after transfection of oeTRIM37 into HI299 cells. **** $P<0.00$ I vs oeNC.

Abbreviation: BLANK, blank control.

of TRIM37 was observed in oeTRIM37-transfected cells. Hence, the oeTRIM37 cells were chosen for the following TRIM37 overexpression analysis.

\section{Suppression of TRIM37 inhibited cell development and metastasis}

The function of TRIM37 in the proliferation of lung cancer cells was examined through CCK-8 assays. siNC, RNAi1-1, and RNAi1-2 were transfected to H358 and H292 cells, respectively. As shown in Figure 3A, there was no obvious difference between BLANK and siNC groups in cell proliferation of the two lung cancer cell lines. However, the cell growth rate of the two cell lines in siTRIM37 group was significantly decreased at 24, 48, and 72 hours compared with siNC group. Thus, these data suggested that TRIM37 is a positive regulator of cell proliferation of lung cancer cells. Next, Annexin V-FITC/propidium iodide staining assay was performed to investigate the effects of TRIM37 on cell apoptosis of lung cancer cells. As shown in Figure 3B, the cell apoptosis ratio of the two cell lines was obviously higher in siTRIM37 group than in siNC group. These result indicated the antiapoptosis function of TRIM37 in lung cancer cells.

Moreover, we also examined the effect of siTRIM37 on lung cancer cells migration and invasion. As shown in Figure 3C and D, both the RNAi1-1 and RNAi1-2 significantly inhibited the migration and invasion ability of H292 or H358 cells. Thus, these data suggested a role for TRIM37 in the promotion of lung cancer cells metastasis.

\section{Knockdown of TRIM37 affected the activity of BCL2, BAX, and AKT}

As shown in Figure $3 \mathrm{E}$ and $\mathrm{F}$, the mRNA and protein expression level of BCL2 was remarkably downregulated in TRIM37 siRNA cells, which was contrary to the expression pattern of BAX. These results were consistent with our expectation. Moreover, the AKT level showed no obvious difference among all transfected cells as indicated above. However, the protein level of p-AKT was highly decreased in siTRIM37 cells, which indicated that the activity of AKT was deeply suppressed when TRIM37 was knocked down. These results might reflect the interaction between AKT and TRIM37 in lung cancer cells.

\section{The function of TRIM37 was inhibited by} a specific PI3K/AKT inhibitor LY294002

A specific AKT inhibitor LY294002 (10 $\mu \mathrm{M})$ was used to further determine the relationship between TRIM37 and AKT. As shown in Figure 4A, the cell proliferation rate of oeTRIM37 was significantly upregulated as compared with that of oeNC cells. However, a remarkable decrease in cell proliferation rate was observed after the cells were treated with LY294002. Moreover, the cell apoptosis rate was significantly inhibited by oeTRIM37 in lung cancer cells. However, 
this suppression effect of TRIM37 was partly reduced after oeTRIM37 cells were treated with LY294002 (Figure 4B). Additionally, the cell migration and invasion ability analysis also showed similar results (Figure 4C).
Western blot was performed to analyze the protein level of BCL2, BAX, AKT, and p-AKT in oeNC, oeNC + LY294002, oeTRIM37, and oeTRIM37 + LY294002 cells, respectively. As shown in Figure 4D, the BCL2 level was

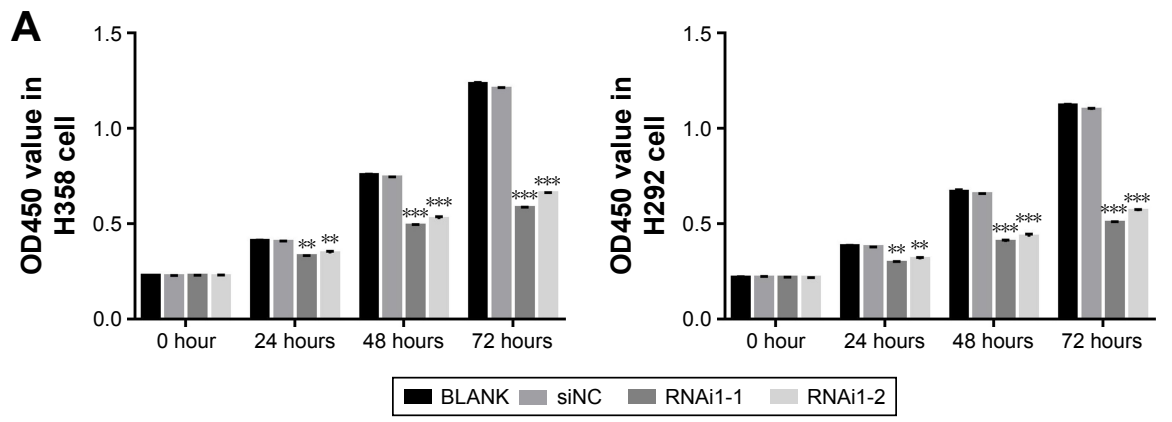

B
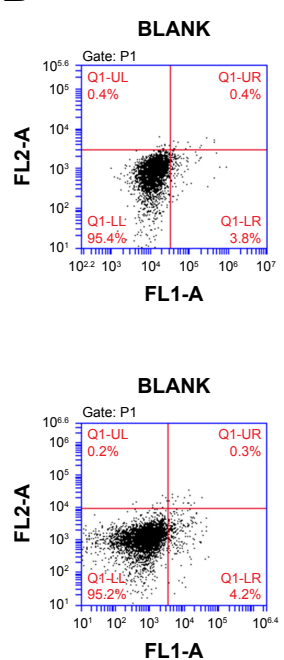

C

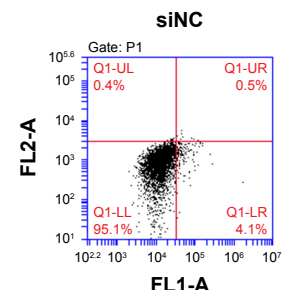

H292

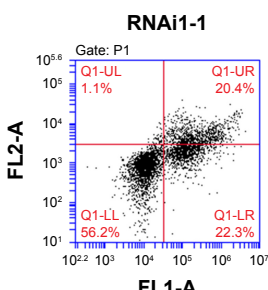

H358

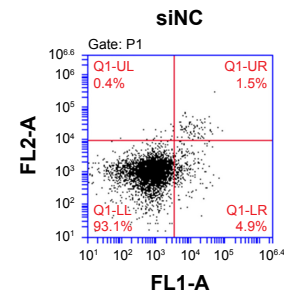

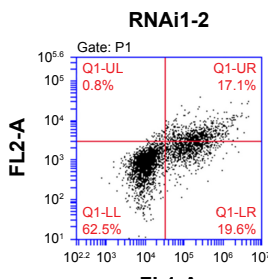

FL1-A

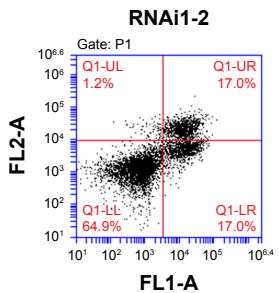

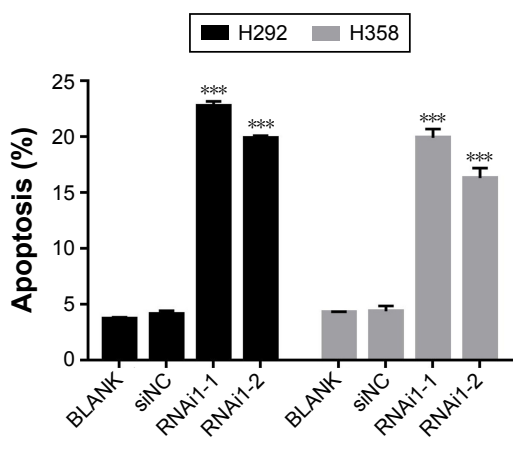

$\mathrm{H} 292$

BLANK

siNC

RNAi1-1

RNAi1-2

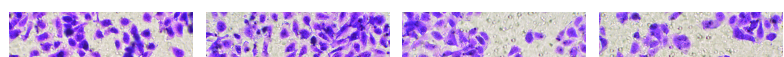

Migration

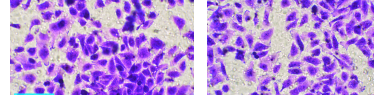

$\rightarrow$ is
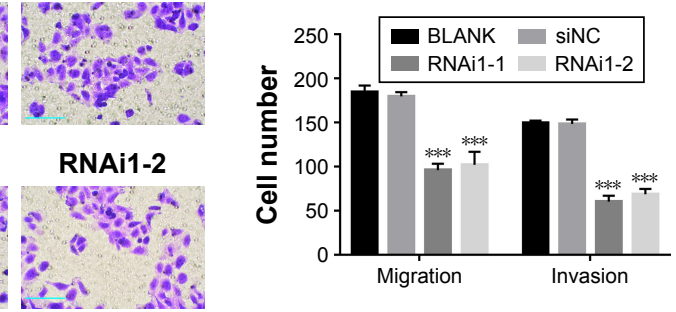

D

H358

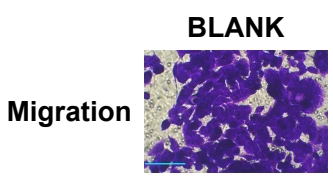

siNC

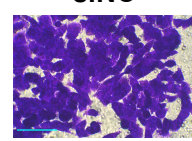

RNAi1-1

RNAi1-2
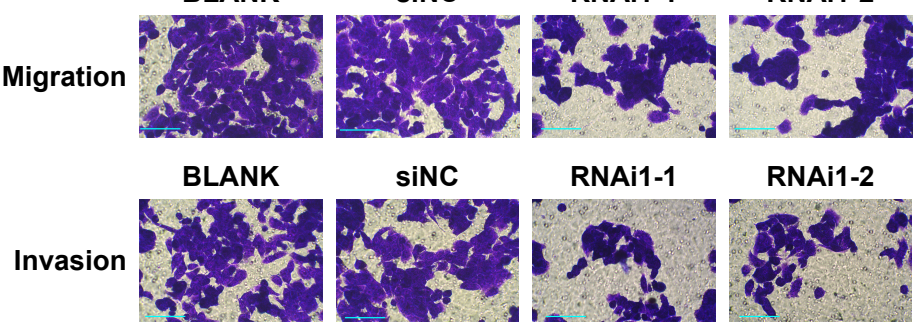

siNC

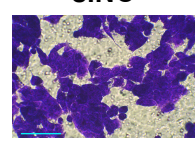

RNAi1-1

RNAi1-2
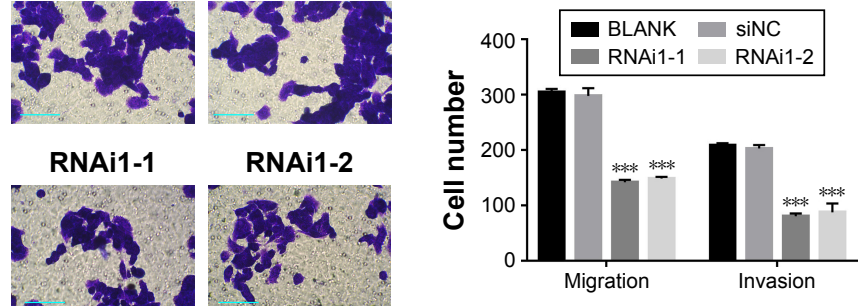

Figure 3 (Continued) 
$\mathrm{E}$
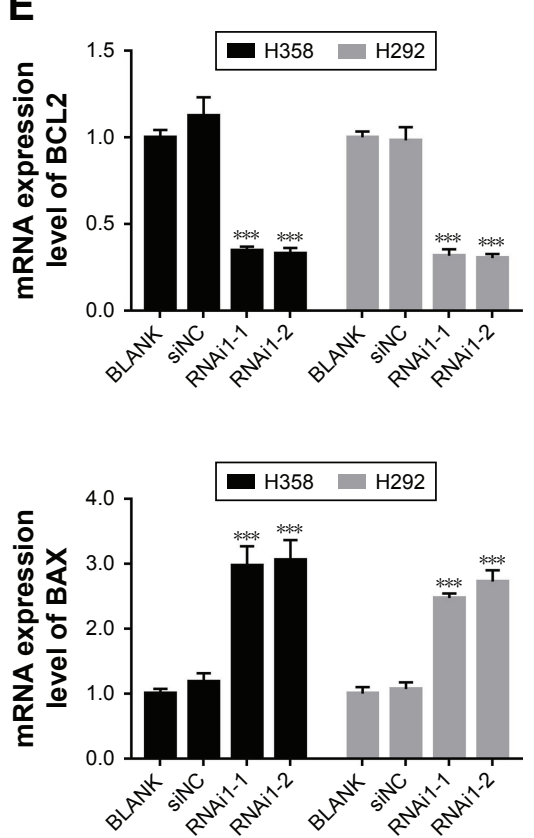

$\mathbf{F}$
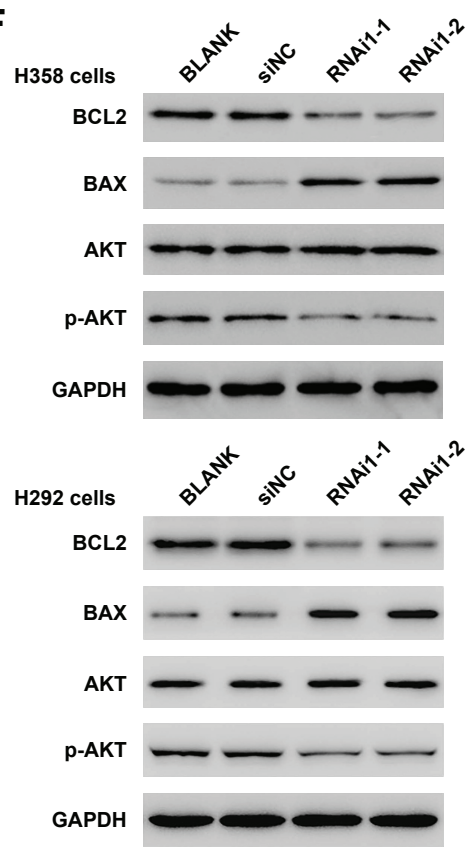
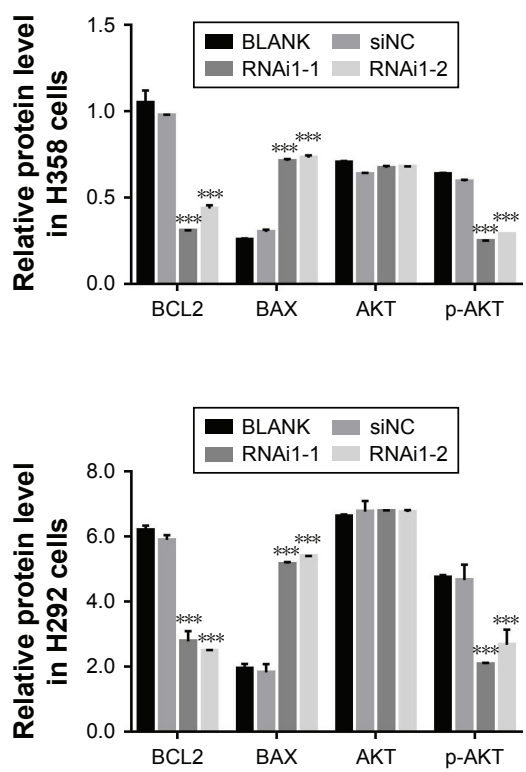

Figure 3 Knockdown of TRIM37 inhibited cell proliferation and apoptosis.

Notes: (A) Cell proliferation was detected at $0,24,48$, and 72 hours after transfection with siNC, siTRIM37I-I, and siTRIM37I-2 in H358 and H292 cells. **P $<0.0$ I vs siNC, and $* * * P<0.00$ I vs siNC. (B) Cell apoptosis profile of cells as indicated above. $* * * P<0.00$ I vs siNC. (C and D) H292 and H358 cells transfected with indicated TRIM37 siRNAs, and cell migration and invasion were analyzed as described in the "Materials and methods" section, respectively. Magnification, $\times 200$. $* * * P<0.00$ I vs siNC group. (E) The mRNA expression level of BCL2 and BAX in cells as indicated above. ${ }^{* * *} P<0.001$ vs siNC. (F) The protein level of BCL2, BAX, AKT, and $p-A K T$ in cells as indicated above. ${ }^{*} * * P<0.001$ vs siNC.

Abbreviation: BLANK, blank control.

highly increased in oeTRIM37 cells as compared with oeNC cells. However, the protein level of BCL2 was significantly downregulated after the oeNC and oeTRIM37 cells were treated with LY294002. The protein level of BAX showed the opposite expression pattern to BCL2. Moreover, the AKT level showed no significant difference among the cells. However, the protein level of p-AKT was deeply inhibited after the cells were treated with LY294002, which suppressed the activity of AKT. Taken together, these results demonstrated that AKT might be a target of TRIM37 in lung cancer cells.

\section{Knockdown of TRIM37 inhibited tumorigenicity of lung cancer cells in vivo}

In order to investigate the function of TRIM37 in tumorigenicity in vivo, an equal number of $\mathrm{H} 358$ cells transfected with siRNA and siTRIM37 were hypodermically injected into nude mice ( $\mathrm{n}=6$ for each group) and tumor formation was determined every 3 days for 33 days (starting at day 12). As shown in Figure $5 \mathrm{~A}$ and $\mathrm{B}$, although both the injected cells were capable of developing tumors, it was easily identified that the TRIM37-siRNA cells significantly suppressed the tumor growth rate. The volume and weight of TRIM37-siRNA tumors showed highly significant reduction as compared with that of siNC tumors. In addition, the siTRIM37-transfected cells contributed to prolong the survival length of nude mice (Figure 5C).

\section{Discussion}

Lung cancer is a highly invasive, rapidly metastasizing, and prevalent cancer all over the world. ${ }^{1}$ Much attention has been paid to explore the effective methods for its treatment. Although the tumorigenic mechanism of lung cancer is still not fully understood, some factors have been found to have high value as biomarkers for diagnosing it at an early stage.

In this study, we systematically analyzed the function of a positive tumor regulator TRIM37 in lung cancer cells. To obtain reliable results, RNA interference was used to silence the expression of TRIM37. On the other hand, lentiviral vector was used to mediate overexpression of TRIM37. The results from those two analyses were consistent, which showed that our results were reliable.

The CCK-8 assay suggested TRIM37 as a positive regulator of cell proliferation of lung cancer cells. A previous report indicated that TRIM37 mediated cell proliferation in pediatric osteosarcoma. ${ }^{6}$ In addition, TRIM44 and TRIM59 have been reported to promote proliferation of lung cancer cells. ${ }^{17,18}$ Therefore, we suspected that all the TRIM proteins contribute to proliferation of lung cancer cells. 
A

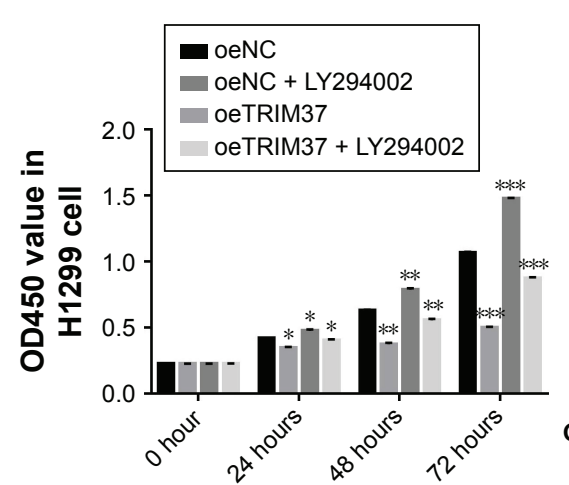

B

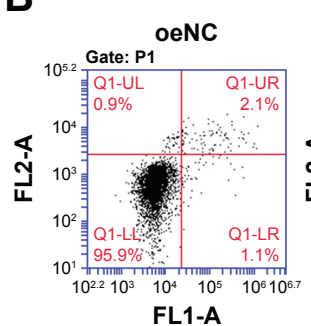

FL1-A

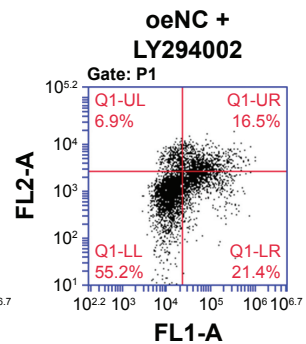

FL1-A
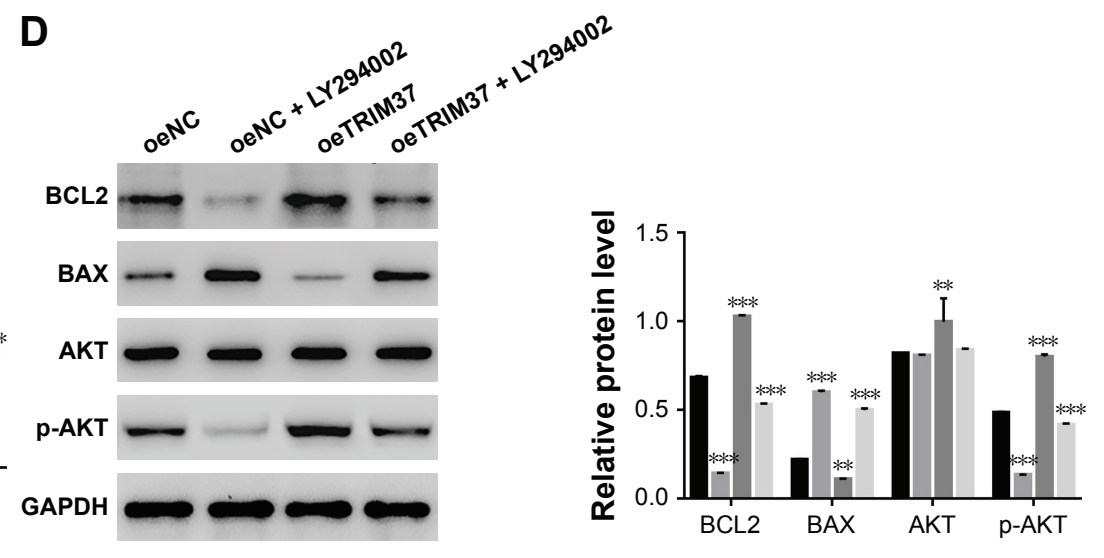

C

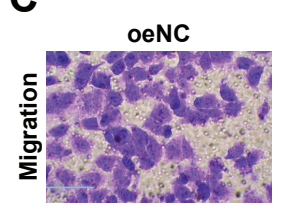

oeNC + LY294002

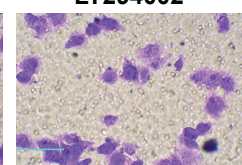

oeNC +

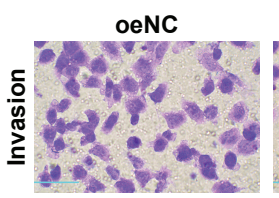
LY294002

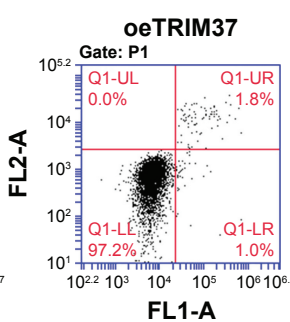

FL1-A
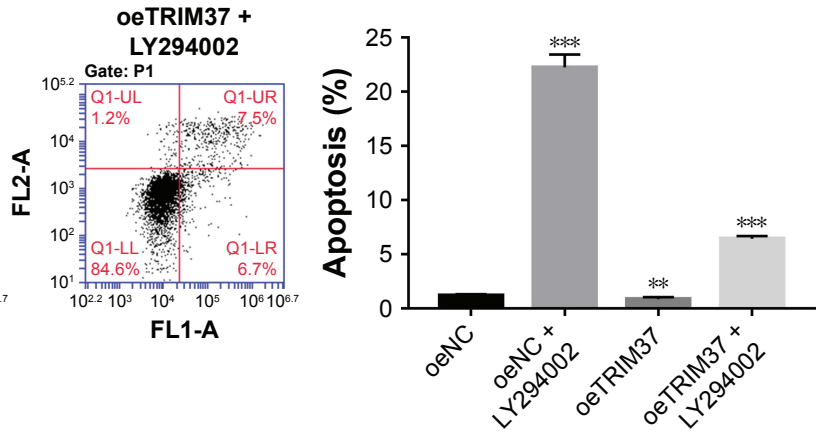

FL1-A
oeTRIM37 +

oeTRIM37

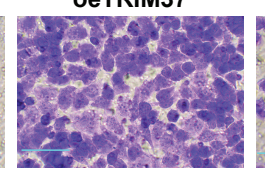

oeTRIM37

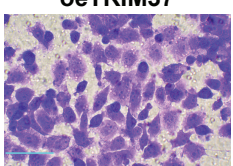

LY294002

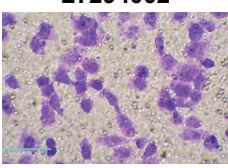

oeTRIM37 + LY294002

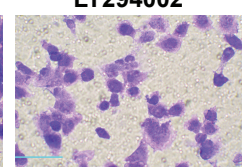

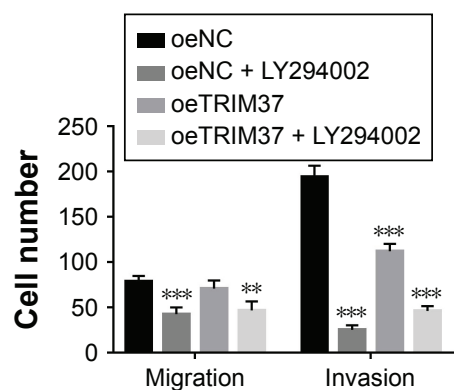

Figure 4 The function of TRIM37 was inhibited by a specific AKT inhibitor LY294002.

Notes: (A) Cell proliferation was detected at 0, 24, 48, and 72 hours after transfection with oeNC, oeNC + LY294002, oeTRIM37, and oeTRIM37 + LY294002 in HI 299 cells. $* P<0.05$ vs oeNC, $* * P<0.01$ vs oeNC, and $* * * P<0.00$ I vs oeNC. (B) Cell apoptosis profile of cells as indicated above. $* * P<0.0 \mathrm{I}$ vs oeNC and $* * * P<0.00 \mathrm{I}$ vs oeNC. (C) The cell migration and invasion data of $\mathrm{HI} 299$ cells transfected with oeNC and oeTRIM37 as well as treated with LY294002. $* * P<0.0 \mathrm{I}$ vs oeNC and $* * * P<0.00 \mathrm{I}$ vs oeNC. (D) The protein level of BCL2, BAX, AKT, and $\mathrm{p}-\mathrm{AKT}$ in cells as indicated above. ${ }^{*} \mathrm{P}<0.0 \mathrm{I}$ vs oeNC and $* * \mathrm{P}<0.00 \mathrm{I}$ vs oeNC.

A

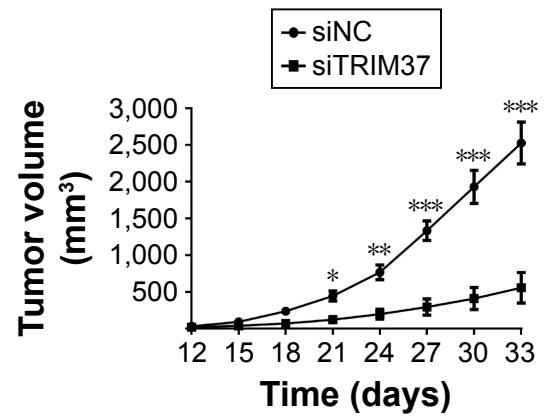

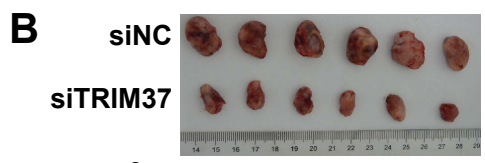

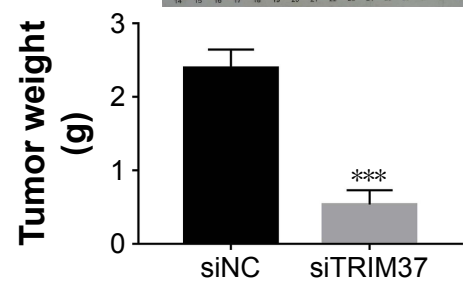

C

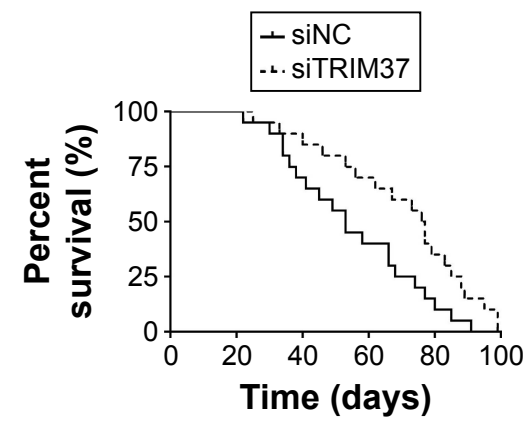

Figure 5 Knockdown of TRIM37 in lung cancer cells inhibited tumor growth in vivo.

Notes: $\mathrm{H} 358$ cells transfected with siRNA control (siNC) or siTRIM37 were subcutaneously injected into athymic nude mice. (A and B) The tumor volume and weight in nude mice injected with $\mathrm{H} 358$ cells transfected with siNC and siTRIM37. $* P<0.05$ vs siNC, $* * P<0.0$ I vs siNC, and $* * * P<0.00$ I vs siNC. (C) The survival rate of nude mice injected with $\mathrm{H} 358$ cells transfected with siNC and siTRIM37. 
A previous study showed that knockdown of TRIM9 induced apoptosis of lung cancer cells through mediating BCL2 and other related proteins..$^{19}$ In the present study, the cell apoptosis results indicated the antiapoptosis function of TRIM37 in lung cancer cells. Moreover, our results showed that the protein level of a pair of cell apoptosis factors (BCL2 and BAX) was deeply influenced when TRIM37 was knocked down or overexpressed in lung cancer cells, which indicates that TRIM37 can serve as an apoptotic marker of primary lung cancer. ${ }^{20} \mathrm{Hence}$, we suspected that the antiapoptosis function of TRIM37 was mainly through the regulation of BCL2 and BAX. Moreover, we also evaluated the function of TRIM37 in cell migration and invasion in lung cancer cells. Our results indicated that the level of TRIM37 was positively correlated with the ability of migration and invasion of lung cancer cells. Previous reports have demonstrated that TRIM37 promoted cell metastasis in pancreatic cancer cells..$^{5,21}$ Our results also insisted this conclusion, which suggested that TRIM37 is a positive regulator of cell metastasis.

It has been reported that TRIM11 functions in lung cancer cells via enhancing the activity of PI3K/AKT. ${ }^{22}$ Previous reports have demonstrated that the oncogenes TRIM22 and TRIM44 contribute to the activity of P13/AKT in lung cancer cells. ${ }^{23,24}$ Moreover, knockdown of TRIM37 in glioma cells led to the inactivation of PI3K/AKT signaling pathway. ${ }^{16}$ In this study, our results indicated that TRIM37 could activate PI3K/AKT. Therefore, we conclude that TRIM37 may promote lung cancer cell development through PI3K/AKT signaling pathway.

Moreover, some evidence has suggested that the activity of AKT is vital for retaining BAX in the cytoplasm. ${ }^{25,26}$ In addition, the AKT protein also functions to upregulate the expression of BCL2. ${ }^{27,28}$ Therefore, we suggest that TRIM37/ AKT/BCL2 pathway might be a novel target for regulating cell apoptosis in lung cancer cells.

\section{Conclusion}

In the present study, we examined the effect of TRIM37 knockdown and overexpression in lung cancer cells. Our results not only help in better understanding of TRIM37 in lung cancer but also provide evidence that TRIM37 might serve as a novel biomarker in clinical application.

\section{Acknowledgments}

This study was supported by the National Natural Science Foundation of China (No 81401943). Shumin Dong and Xueqin Pang are co-first authors for this study.

\section{Disclosure}

The authors report no conflicts of interest in this work.

\section{References}

1. Lemjabbar-Alaoui H, Hassan OU, Yang YW, Buchanan P. Lung cancer: Biology and treatment options. Biochim Biophys Acta. 2015; 1856(2):189-210.

2. Alberg AJ, Brock MV, Ford JG, Samet JM, Spivack SD. Diagnosis and Management of Lung Cancer, 3rd ed: American College of Chest Physicians Evidence-Based Clinical Practice Guidelines. Epidemiology of Lung Cancer. 2016;143(5).

3. Kallijärvi J, Lahtinen U, Hämäläinen R, Lipsanen-Nyman M, Palvimo JJ, Lehesjoki AE. TRIM37 defective in mulibrey nanism is a novel RING finger ubiquitin E3 ligase. Exp Cell Res. 2005;308(1):146-155.

4. Hu CE, Gan J. TRIM37 promotes epithelial-mesenchymal transition in colorectal cancer. Mol Med Rep. 2017;15(3):1057-1062.

5. Jiang J, Tian S, Yu C, Chen M, Sun C. TRIM37 promoted the growth and migration of the pancreatic cancer cells. Tumour Biol. 2016;37(2): 2629-2634.

6. Tao Y, Xin M, Cheng H, et al. TRIM37 promotes tumor cell proliferation and drug resistance in pediatric osteosarcoma. Oncol Lett. 2017;14(6): 6365-6372.

7. Viant C, Guia S, Hennessy RJ, et al. Cell cycle progression dictates the requirement for BCL2 in natural killer cell survival. J Exp Med. 2017; 214(2):491-510.

8. Cui J, Placzek WJ. Post-Transcriptional Regulation of Anti-Apoptotic BCL2 Family Members. Int J Mol Sci. 2018;19(1):E308.

9. Sun SY, Yue P, Zhou JY, et al. Overexpression of BCL2 blocks TNF-related apoptosis-inducing ligand (TRAIL)-induced apoptosis in human lung cancer cells. Biochem Biophys Res Commun. 2001;280(3): 788-797.

10. Gessner C, Liebers U, Kuhn H, et al. BAX and p16INK4A are independent positive prognostic markers for advanced tumour stage of nonsmall cell lung cancer. Eur Respir J. 2002;19(1):134-140.

11. Han B, Park D, Li R, et al. Small-Molecule Bcl2 BH4 Antagonist for Lung Cancer Therapy. Cancer Cell. 2015;27(6):852-863.

12. Chen MJ, Wu DW, Wang GC, Wang YC, Chen CY, Lee H. MicroRNA630 may confer favorable cisplatin-based chemotherapy and clinical outcomes in non-small cell lung cancer by targeting Bcl-2. Oncotarget. 2018;9(17):13758-13767.

13. Yoshizawa A, Fukuoka J, Shimizu S, et al. Overexpression of phosphoeIF4E is associated with survival through AKT pathway in non-small cell lung cancer. Clin Cancer Res. 2010;16(1):240-248.

14. Vincent EE, Elder DJ, Thomas EC, et al. Akt phosphorylation on Thr308 but not on Ser473 correlates with Akt protein kinase activity in human non-small cell lung cancer. Br J Cancer. 2011;104(11): 1755-1761.

15. Xu X, Zhang Y, Qu D, Jiang T, Li S. Osthole induces G2/M arrest and apoptosis in lung cancer A549 cells by modulating PI3K/Akt pathway. J Exp Clin Cancer Res. 2011;30:33.

16. Tang SL, Gao YL, Wen-Zhong H. Knockdown of TRIM37 suppresses the proliferation, migration and invasion of glioma cells through the inactivation of PI3K/Akt signaling pathway. Biomed Pharmacother. 2018;99:59-64.

17. Xing Y, Meng Q, Chen X, et al. TRIM44 promotes proliferation and metastasis in non-small cell lung cancer via mTOR signaling pathway. Oncotarget. 2016;7(21):30479-30491.

18. Zhan W, Han T, Zhang C, et al. TRIM59 Promotes the Proliferation and Migration of Non-Small Cell Lung Cancer Cells by Upregulating Cell Cycle Related Proteins. PLoS One. 2015;10(11):e0142596.

19. Wang X, Shu Y, Shi H, Lv X, Zou H, Shi W. TRIM9 is up-regulated in human lung cancer and involved in cell proliferation and apoptosis. Int J Clin Exp Med. 2016;9(6):10461-10469.

20. Porebska I, Wyrodek E, Kosacka M, Adamiak J, Jankowska R, Harłozińska-Szmyrka A. Apoptotic markers p53, Bcl-2 and Bax in primary lung cancer. In Vivo. 2006;20(5):599-604.

21. Jiang J, Yu C, Chen M, Tian S, Sun C. Over-expression of TRIM37 promotes cell migration and metastasis in hepatocellular carcinoma by activating Wnt/ $\beta$-catenin signaling. Biochem Biophys Res Commun. 2015;464(4):1120-1127. 
22. Wang X, Shi W, Shi H, et al. TRIM11 overexpression promotes proliferation, migration and invasion of lung cancer cells. J Exp Clin Cancer Res. 2016;35(1):100.

23. Liu L, Zhou XM, Yang FF, et al. TRIM22 confers poor prognosis and promotes epithelial-mesenchymal transition through regulation of AKT/ GSK3 $\beta / \beta$-catenin signaling in non-small cell lung cancer. Oncotarget. 2017;8(37):62069-62080

24. Xing Y, Meng Q, Chen X, Wang X, et al. TRIM44 promotes proliferation and metastasis in non-small cell lung cancer via mTOR signaling pathway. Oncotarget. 2016;7(21):30479-30491.

25. Tsuruta F, Masuyama N, Gotoh Y. The phosphatidylinositol 3-kinase (PI3K)-Akt pathway suppresses Bax translocation to mitochondria. J Biol Chem. 2002;277(16):14040-14047.
26. Yamaguchi H, Wang HG. The protein kinase PKB/Akt regulates cell survival and apoptosis by inhibiting Bax conformational change. Oncogene. 2001;20(53):7779-7786.

27. Marinov M, Ziogas A, Pardo OE, et al. AKT/mTOR pathway activation and BCL-2 family proteins modulate the sensitivity of human small cell lung cancer cells to RAD001. Clin Cancer Res. 2009;15(4): 1277-1287.

28. Pugazhenthi S, Nesterova A, Sable C, et al. Akt/protein kinase B upregulates Bcl-2 expression through cAMP-response element-binding protein. J Biol Chem. 2000;275(15):10761-10766. 


\section{Supplementary materials}

Primer sequence information

Homo sapiens TRIM37, transcript variant 2, mRNA

NCBI Reference Sequence: NM_001005207.4

Primer F: 5' TGGACTTACTCGCAAATG 3'

Primer R: $5^{\prime}$ ATCTGGTGGTGACAAATC $3^{\prime}$

Pos: 1645-1874 C

Amplified product: size: 230 bps, product GC: $39 \%$

H. sapiens BCL2, apoptosis regulator, transcript variant alpha, mRNA

NCBI Reference Sequence: NM_000633.2

Primer F: 5' GCAGTGTGGTCTCCGAATGTC 3'

Primer R: 5' CATTGCCTCTCCTCACGTTCC 3'

Pos: 2277-2521 C

Amplified product: size: 245 bps, product GC: $56 \%$
H. sapiens $B A X$, apoptosis regulator, transcript variant I, mRNA

NCBI Reference Sequence: NM_001291428.1

Primer F: 5' CTGAGCGAGTGTCTCAAG 3'

Primer R: 5' CAGCCCATGATGGTTCTG 3'

Pos: 244-485 C

Amplified product: size: 242 bps, product GC: $57 \%$

H. sapiens GAPDH, transcript variant I, mRNA

NCBI Reference Sequence: NM_001256799.2

Primer F: 5' AATCCCATCACCATCTTC 3'

Primer R: 5' AGGCTGTTGTCATACTTC 3'

Pos: 436-653

Amplified product: size: 218 bps

Table SI Homo sapiens TRIM37 (NM_00 I005207.4) RNAi targeting locus information

\begin{tabular}{|c|c|c|}
\hline \multicolumn{2}{|c|}{ RNAi targeting locus } & \multirow[t]{2}{*}{ Sequence } \\
\hline Name & Locus position (bp) & \\
\hline RNAil-I & $595-613$ & GCTCCAAACTGTGTTGTTT \\
\hline RNAil-2 & $968-986$ & GGAACTGATCAGCTTAGTT \\
\hline RNAil-3 & $2,501-2,519$ & GCGAGTGCCCTCTGATTTA \\
\hline
\end{tabular}

Table S2 The primary antibodies information

\begin{tabular}{l|l|l}
\hline Antibody name & Source & Dilution factor \\
\hline TRIM37 & CST, Danvers, MA, USA & $1: 1,000$ \\
BCL2 & Abcam, Cambridge, UK & $1: 1,000$ \\
BAX & Abcam & $1: 1,000$ \\
AKT & CST & $1: 1,000$ \\
P-AKT & CST & $1: 2,000$ \\
GAPDH & CST & $1: 2,000$ \\
\hline
\end{tabular}

OncoTargets and Therapy

\section{Publish your work in this journal}

OncoTargets and Therapy is an international, peer-reviewed, open access journal focusing on the pathological basis of all cancers, potential targets for therapy and treatment protocols employed to improve the management of cancer patients. The journal also focuses on the impact of management programs and new therapeutic agents and protocols on patient perspectives such as quality of life, adherence and satisfaction. The manuscript management system is completely online and includes a very quick and fair peer-review system, which is all easy to use. Visit http://www.dovepress.com/testimonials.php to read real quotes from published authors. 